\title{
Mechanical Monitoring Issues in Preparation to Next Step of Wendelstein 7-X Operation
}

\author{
Victor Bykov, André Carls, Jiawu Zhu, Paul van Eeten, Lutz Wegener, Hans-Stephan Bosch, and \\ W7-X team
}

This work has been carried out within the framework of the EUROfusion Consortium and has received funding from the Euratom research and training program 2014-2018 under Grant 633053.

The authors are with the Max Planck Institute for Plasma Physics, EURATOM Association, Greifswald

17491, Germany (e-mail: victor.bykov@ipp.mpg.de; (Corresponding author)

andre.carls@ipp.mpg.de; jiawu.zhu@ipp.mpg.de; paul.van.eeten@ipp.mpg.de;

lutz.wegener@ipp.mpg.de; bosch@ipp.mpg.de).

\section{Abstract}

The largest modular stellarator Wendelstein 7-X (W7-X) has successfully passed the first phase of operation in Greifswald, Germany. The sophisticated W7-X superconducting magnet system (MS) with its nonlinear support system has been carefully monitored using an extensive set of mechanical and temperature sensors. This paper focuses on detailed consideration of cyclic MS behavior during operation with limiter configurations of plasma. Measurement results are carefully compared with predictions from updated numerical models and critical issues are highlighted. As a result, the structural monitoring tool is extended to follow enhanced requirements and expectations. This paper is a preparation for upcoming more demanding phases with longer plasma pulses to guarantee safe and reliable W7-X operation with different divertor and scraper element configurations. The procedure to establish required sensor configurations, and to analyze and release new plasma regimes being compatible with $\mathrm{W} 7-\mathrm{X}$ component design values is also described.

\section{Index Terms}

Numerical finite-element (FE) analysis, stellarator, structural monitoring, Wendelstein 7-X (W7-X).

\section{INTRODUCTION AND MOTIVATION}

The largest modular stellarator Wendelstein 7-X (W7-X) has successfully entered commissioning process before the next phase of operation (OP1.2a) in Greifswald, Germany. The first phase (OP1.1) with limiter configurations of plasma with $2.5 \mathrm{~T}$ of magnetic induction on the plasma axis met successfully all project team goals. About 940 discharge programs have been done with maximum pulse length up to $6 \mathrm{~s}$ and with maximum input energy of 4 MJ. Besides main physical achievements (see [1]-[3]) it is necessary to mention that considerable generated structural loads (meganewton) in the W7-X systems were successfully endured.

Fivefold symmetric magnet system (MS) comprises 50 nonplanar coil (NPC) and 20 planar coil (PLC) superconducting coils. In addition to the superconducting coils, a set of five normal conducting trim coils (TCs) could be in operation to allow a fine tuning of the main magnetic field. The MS has to provide a wide range of different magnetic field configurations already during coming operation phase (see Table I). Double current levels are planned in planar coils and TCs. The sophisticated W7-X systems require advanced numerical modeling. The main global finite-element (FE) models have been created and improved for the demanding task (see Fig. 1). The main structural components of W7-X are presented schematically in Fig. 1 using few cutouts. Moreover, the complex support structure of the W7-X MS includes a large number of nonlinear components (see Fig. 2). Each superconducting coil is connected to the central support structure by two bolted central support elements (CSEs) allowing possible opening of the flange under high loadings.

The mechanical intercoil support structure encompasses many sliding narrow support elements at the inboard part of all five differently shaped NPCs (type 1-5 coils). The planar coil support elements (PSEs) connect two types of PLC (types A and B) to NPC types 2, 3 and types 4, 5, respectively. One PSE per coil (PSE-A1 to NPC 
type 2 and PSE-B1 to NPC type 5) is a fixed bolted connection, while other PSEs are sliding as the narrow supports. The intermodule lateral supports at outboard part are also bolted connections and only ones inside the module are welded elements. Each MS module consists of two flip-symmetric half modules imposing

"stellarator symmetry." As a result the central support structure is bolted from ten units. Two of ten MS cryolegs fastened to the CSS at the top and sliding/rotating at the bottom bearings installed on machine base are visible in Fig. 1. Toroidal tie-rods fix the position of the MS without restrictions for shrinkage during MS cooldown. Both the dead weight and the cryolegs break the stellarator symmetry and introduce slightly different behavior of half modules.

TABLE I COIL CURRENTS FOR OP1.1 AND OP1.2A OPERATION

\begin{tabular}{|c|c|c|c|c|c|c|c|c|}
\hline \multirow{3}{*}{\multicolumn{2}{|c|}{ Coil type }} & \multicolumn{7}{|c|}{ Coil current, kA } \\
\hline & & \multicolumn{2}{|c|}{ OP1.1/ OP $1.2 \mathrm{a}$} & \multicolumn{5}{|c|}{ Planned (demanding) new 2.5T regimes during OP1.2a (reference cases only) } \\
\hline & & $\begin{array}{l}\text { "J regime" } \\
\text { Limiter (0) }\end{array}$ & $\begin{array}{l}\text { "A regime" } \\
\text { Standard (2) }\end{array}$ & $\begin{array}{l}\text { High Iota } \\
\text { (1) }\end{array}$ & $\begin{array}{l}\text { High mirror } \\
\text { (2) }\end{array}$ & $\begin{array}{c}\text { Low mirror } \\
\text { (2) }\end{array}$ & $\begin{array}{c}\text { Inward } \\
\text { shifted (3) }\end{array}$ & $\begin{array}{c}\text { Low iota } \\
(4)^{*}\end{array}$ \\
\hline 1 & Type 1 & 12.8 & 13.5 & 14.9 & 14.5 & 12.6 & 13.1 & 12.2 \\
\hline 2 & Type 2 & 12.8 & 13.5 & 14.9 & 14.1 & 13.2 & 13.0 & 12.2 \\
\hline 3 & Type 3 & 12.8 & 13.5 & 14.9 & 13.4 & 13.2 & 13.2 & 12.2 \\
\hline 4 & Type 4 & 12.8 & 13.5 & 14.9 & 12.8 & 14.2 & 14.6 & 12.2 \\
\hline 5 & Type 5 & 12.8 & 13.5 & 14.9 & 12.4 & 14.2 & 14.7 & 12.2 \\
\hline 6 & Type A & $0 \div 5$ & 0 & -10.3 & 0 & 0 & 4.1 & 9.2 \\
\hline 7 & Type B & $0 \div 5$ & 0 & -10.3 & 0 & 0 & -8.2 & 9.2 \\
\hline \multicolumn{2}{|c|}{ Outer Trim coils } & 1.1 & & $1.8 / 1.95$ & $1.8 / 1.95$ & $1.8 / 1.95$ & $1.8 / 1.95$ & $1.8 / 1.95$ \\
\hline
\end{tabular}

* Index indicates polarities of planar coils

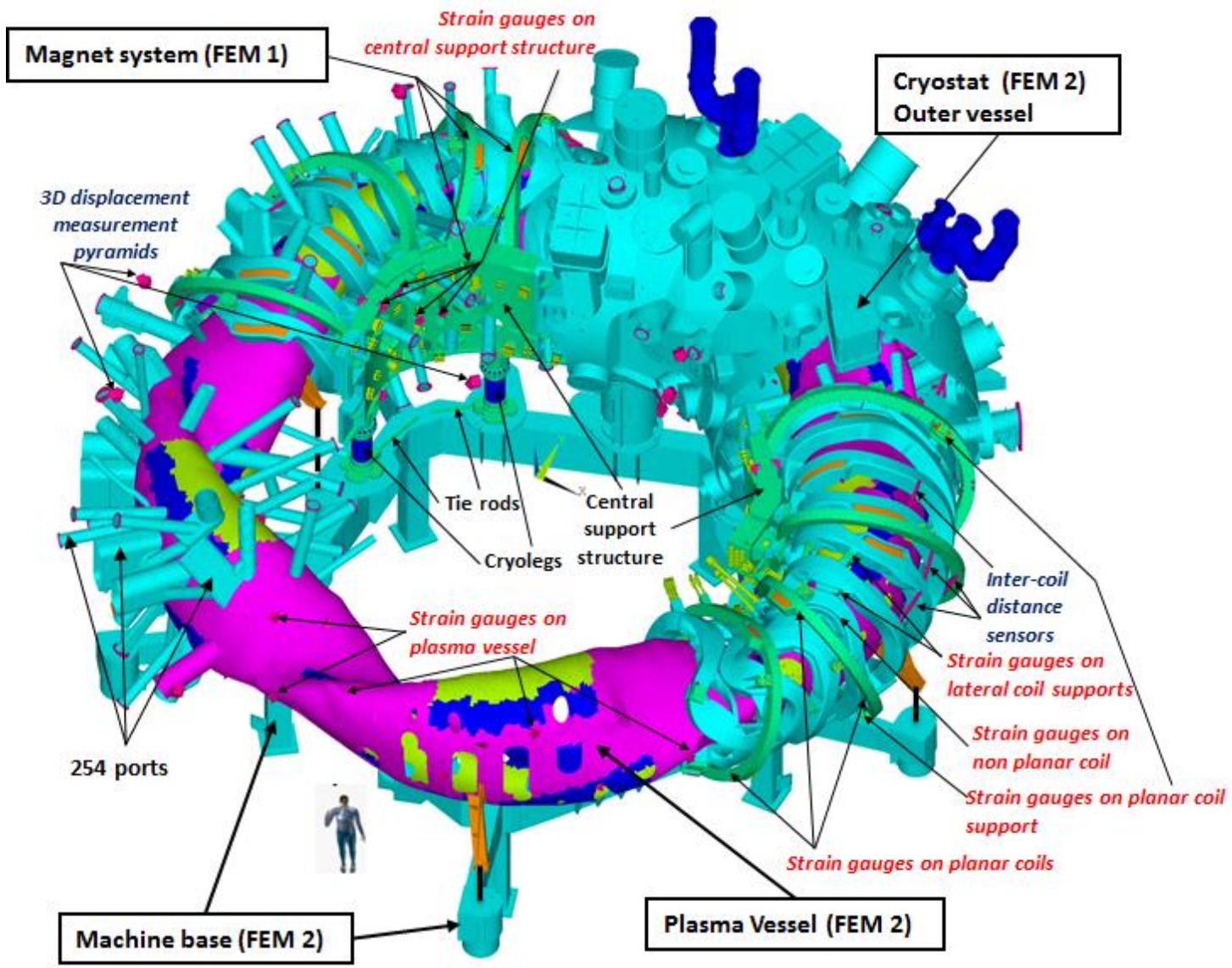

Fig. 1. Fragment of global FE models of cryostat [6] and MSs with identification of typical sensor positions.

In order to assess the mechanical integrity of the MS during design, assembly, commissioning, and first phase of operation, two global FE models in ANSYS and ABAQUS were intensely used and benchmarked [4]. The large amount of nonlinear components in the MS makes its behavior prediction challenging.

Other complications are the elements with different orders of stiffness and the fact that nonlinear geometry option is to be activated to get accurate results. The present strategy is to migrate to one improved ANSYS global model (GM), which is suitable to deliver reliable FE predictions. 
In addition, more than 200 detailed local FE analyses of critical components have been performed to confirm that all elements are within their structural limits. Such local FE models also allow study of behavior of the critical supports under maximum loadings and beyond.

The unique feature of W7-X is a mechanical instrumentation (MI) system with roughly 800 sensors. The system allows to confirm advanced calculation approaches and results, as well as to guarantee safe operation. In addition to the mechanical data, temperatures, current levels, and hydraulic parameters, (altogether more than 3000

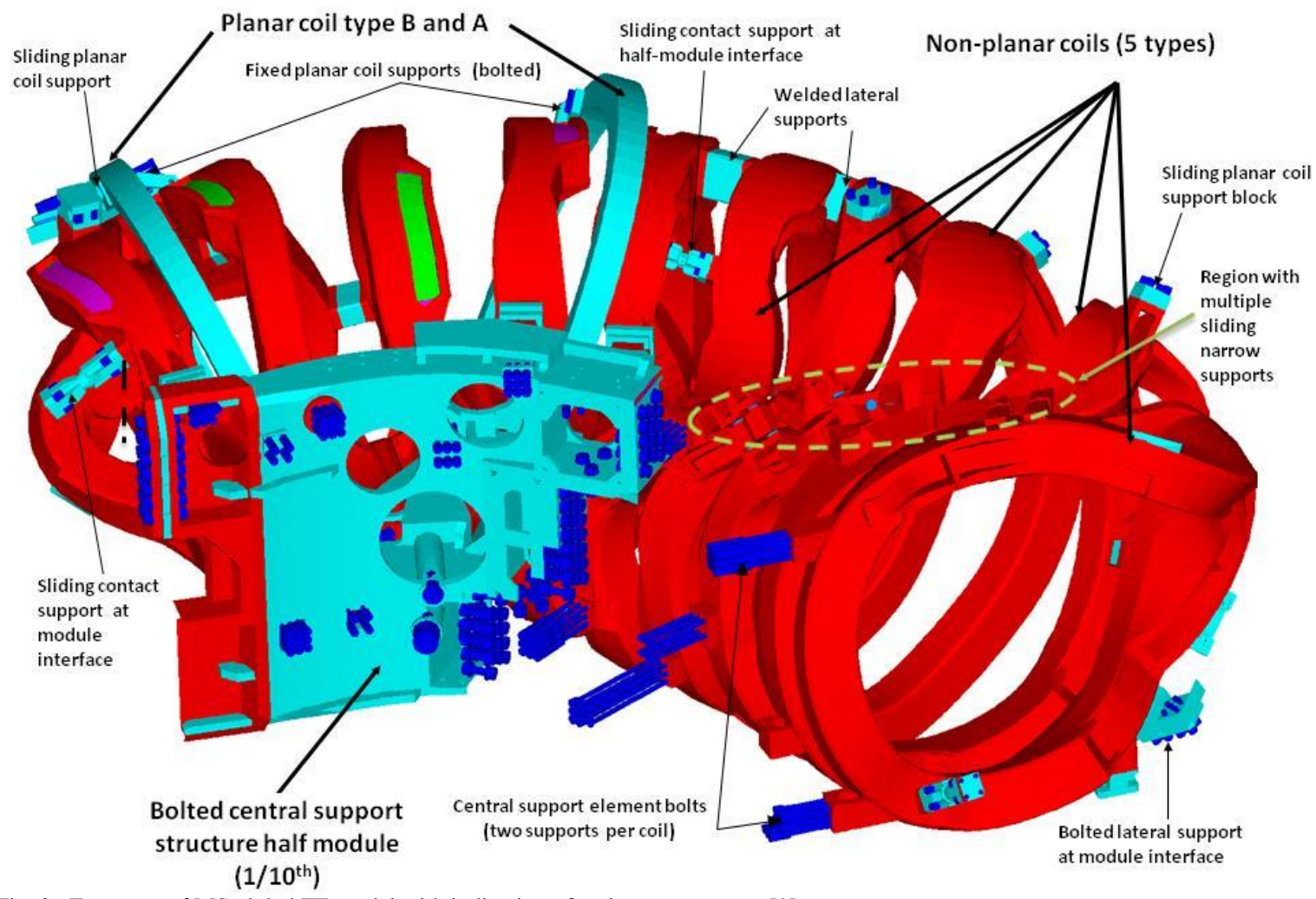

Fig. 2. Fragment of MS global FE model with indication of main support types [9].

signals) are also collected. Their simultaneous monitoring and careful evaluations are the key issue during device commissioning and operation.

The coming project team goals are the physics programs for the operation phase OP1.2a (August 2017December 2018) with an inertially cooled divertor, much higher input energy (up to $80 \mathrm{MJ}$ ) and longer pulses (up to $60 \mathrm{~s}$ ).

\section{MECHANICAL MONITORING}

\section{A. General Remarks}

The direct monitoring of critical components and benchmarking of signals against the results of the nonlinear numerical models is the only way to ensure safe operation of the machine. Achievement of the above-mentioned goal is only possible with close interlink between the MI and numerical models.

The MI is described in detail in [5]. Three main groups of the MI instrumentation were installed in W7-X: strain, distance change, and contact sensors (see Fig. 1).

The primary goal of the global FE models is to predict the stiffnesses and displacements of components, which could affect either plasma equilibrium properties [7] or heat loads on in-vessel components. Therefore, displacement sensors are of great importance. Two ranges of displacements (up to $3 \mathrm{~mm}$ and up to $20 \mathrm{~mm}$, respectively) were originally specified by FE analysis. There are 70 of such latter devices installed in the machine in different locations with different directions (see Figs. 1 and 3).

Apart from displacement measurements of cold components of the MS, also the 3-D-movements of all ten MS weight supports (cryolegs [8]) need to be monitored. A special 3-D displacement measurement unit of pyramid type has been developed and installed at the (warm) bottom of the cryoleg [5].

Possible contacts between the MS and the cryostat in the critical narrow-gap areas could be detected by an electrical short due to contact between two electrically insulated stainless steel foils. During commissioning and first phase operation no confirmed contacts were detected. 
The number and locations of strain gauges (SGs) on the coils were determined before start of coil production when the final loads were not yet known in all details. So some of these sensors are not on optimal positions but still yield valuable information. The locations on the structure elements were determined later with extended

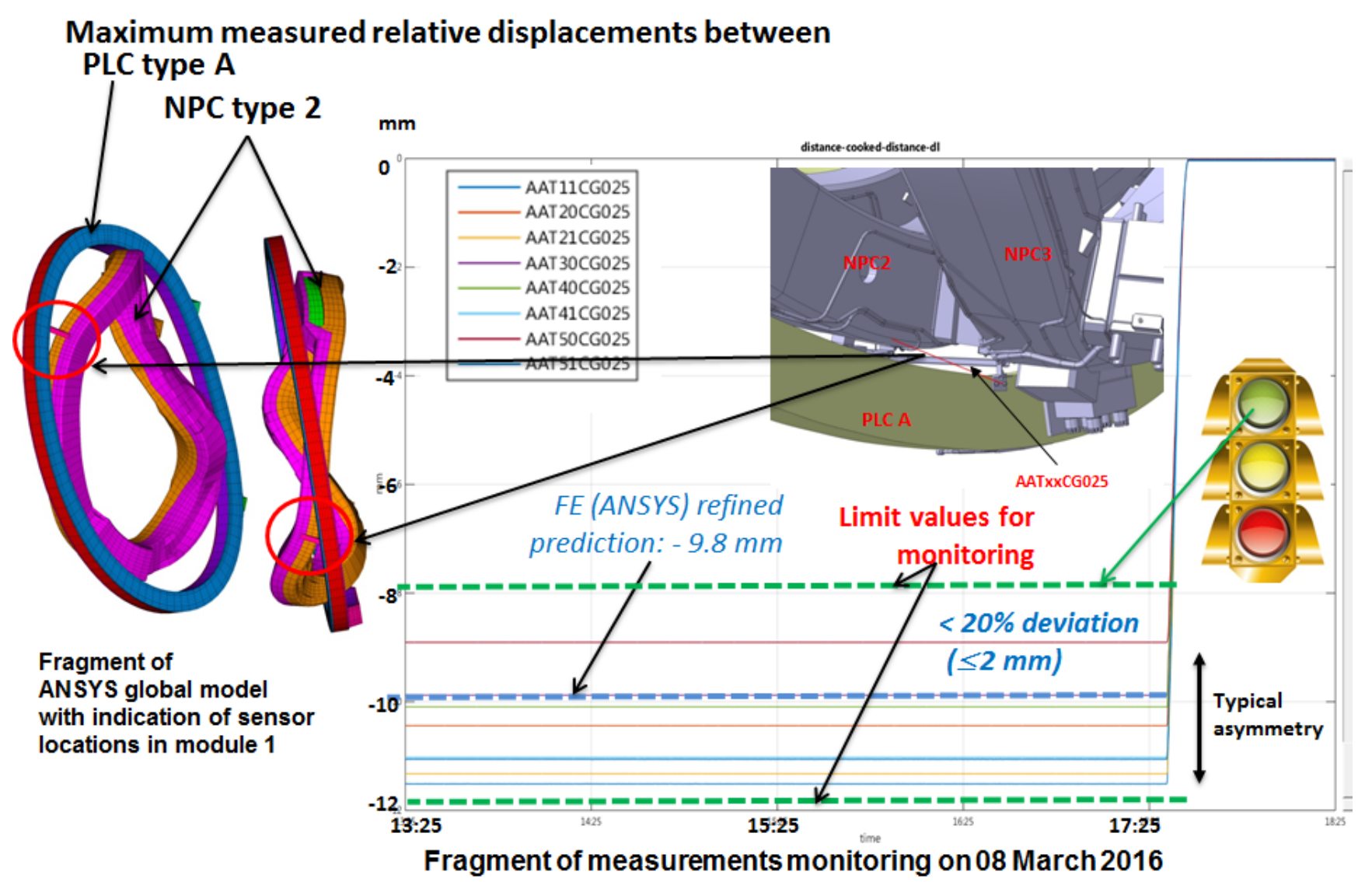

Fig. 3. Results of monitoring for maximum coils mutual displacements between NPC type 2 coils and PLC type A coils. Typical set-up for the wire sensors extracted from CATIA model.

knowledge about critical components. First priority was given to instrumentation of Inconel bolts of CSE connections (see fragment in Fig. 4) under bending and tension, critical region with expected yielding on each half module of the CSS and in the corners of coil blocks for bolted lateral support bridges [9]. Other SGs are located in areas with moderate stress, mainly intended to support the FE model benchmarking.

Part of the MI sensors are distributed symmetrically over the five W7-X modules and the others asymmetrically in order to check the MS symmetry and to cover more locations, respectively.

Strong temperature dependence of the SG resistance jeopardizes the measurements at the temperature region below $10 \mathrm{~K}$; therefore, special compensation procedure is to be implemented [9].

\section{B. Monitoring Approach}

Due to MS complex structural design and a limited life time, each step of W7-X commissioning and operation is carefully monitored by a considerable amount of different sensors. Prior to the loading step a set of the boundary or limit values for MI signals are established to distinguish nominal, questionable, and critical state of the machine [10], [11], as also indicated in Fig. 3.

Real-time monitoring of multiple-sensor signals requires careful choice of the way to display the results. In case of MI, it is absolutely necessary to monitor temperatures in parallel with structural signals to avoid misinterpretation. Two or three levels of views, from minimum/maximum/average values down to the individual sensor signal, are found most appropriate. The mechanical monitoring software (MIViewer) [12] includes also signal filtering and careful signal synchronization. The derived and implemented algorithms are the basis for the current and the future deployments in a service oriented W7-X infrastructure. The program is flexible and could be in use for tasks beyond the initial scope. Many functions of

MIViewer have been extensively used now for signal postprocessing purposes (see Figs. 4 and 5 and Tables II and III). 


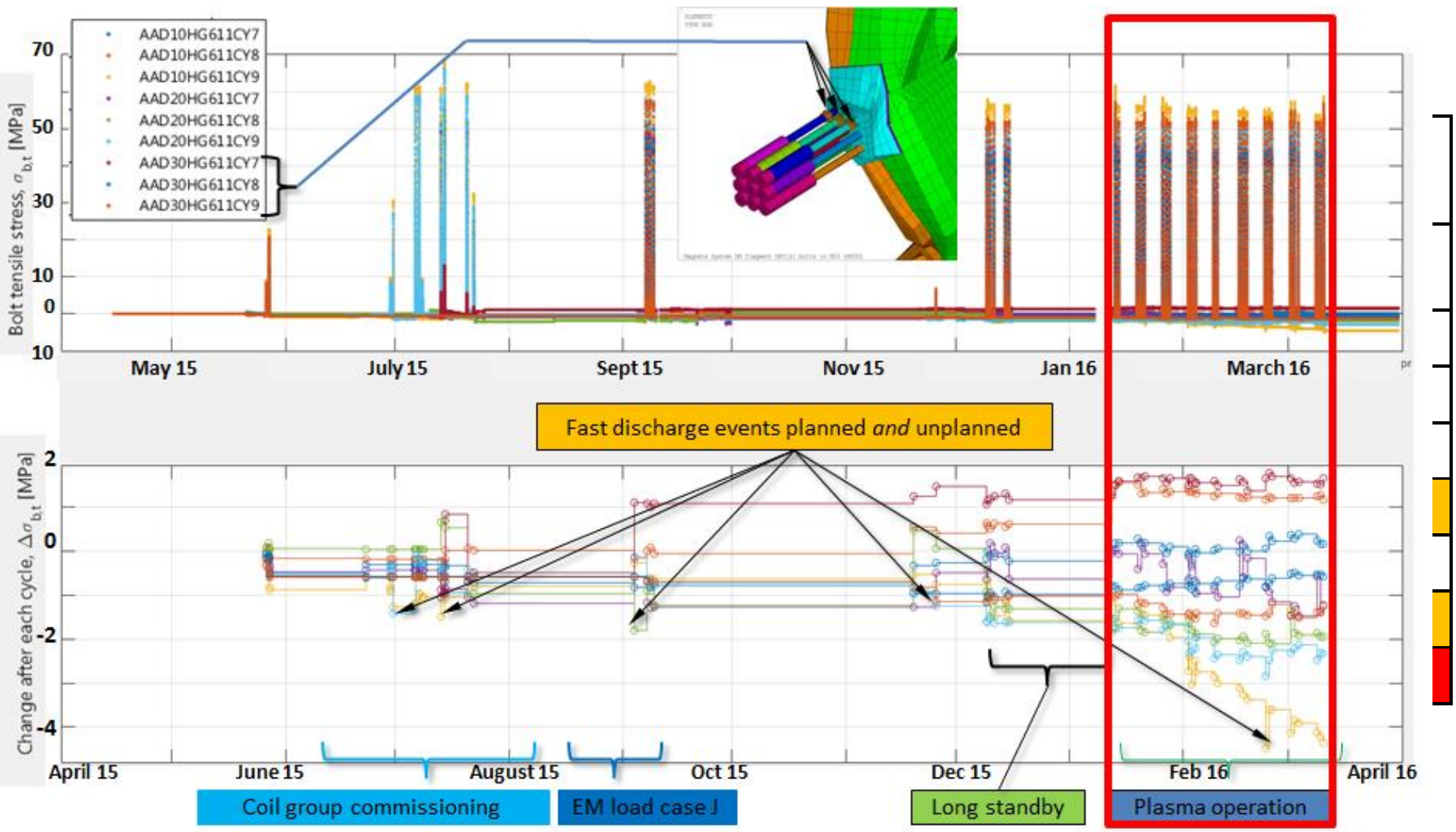

Fig. 4. Detection of all cycles measured in critical bolts (top) and calculation of remaining bolt pretention offsets (bottom). Fragment of FE model presents coil, coil extension, bolts and sleeves as well as strain gauge locations.

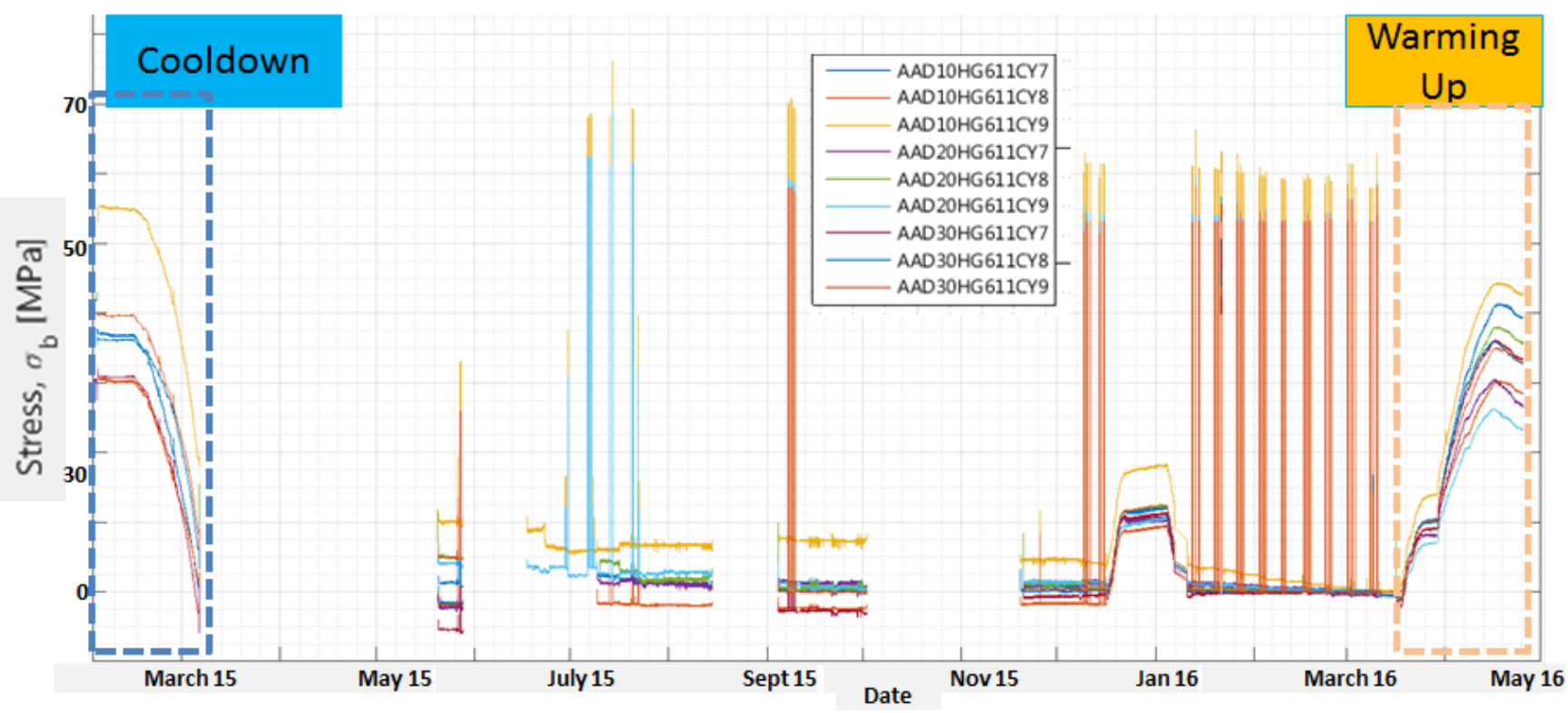

Fig. 5. Estimation of bolt pretention offsets (megapascals) due to cooldown/warming up cycle (see detail values in Table. IV).

However, due to present availability of required electronics only selected set of MI sensor signals is captured and stored in the W7-X experiment archive. Therefore, the approval of the structural integrity by mechanical engineers incorporates also reviews of FE analysis results, especially in case of observed deviations between measurement results and FE predictions. 


\section{OPERATION MONITORING}

\section{A. General Remarks}

All measurement systems have been tested prior the operation and it was found that reliable results are at the level of above $20 \mathrm{MPa}$ and $1 \mathrm{~mm}$ for SGs and mutual displacements between coils, respectively. Measurement pyramids have been more accurate $(0.1-0.5 \mathrm{~mm}$ for different directions) and fully reliable.

Structural integrity of the MS with respect to electromagnetic forces is of main interest. Therefore, the signals had been set to zero when the operation temperature of $\sim 4 \mathrm{~K}$ is reached. Moreover, such zeroing had been done basically each time after temperature increase above $10 \mathrm{~K}$. Later, it was found that with such approach the residual stress could be neglected as well as the residual displacements, which are below $0.5 \mathrm{~mm}$ with exceptions for cryoleg sliding. However, originally implemented zeroing process did not care about winter/summer time shift; therefore it was decided to create a special signal zeroing stream to avoid the problem, to accelerate signal postprocessing and to guarantee repetitive procedure.

\section{PREPARATION FOR NEXT PHASE OF OPERATION}

\section{A. General}

The coming operation phase with an inertial cooled divertor allows much higher input energy and longer pulse. Many engineering challenges have been resolved to provide the abilities to achieve the goal [14].

Multiple-operation cycles with higher energy could significantly increase the temperature of plasma vessel, parts of passively cooled ports, and in-cryostat bellows. It is expected that much higher heat flux is to be delivered on the cryogenically cooled structures with corresponding increasing of temperature by few degrees. This results in considerable SG signal changes. In order to avoid false alarm and to guarantee smooth operation, it is necessary to implement a compensation of the effect using signals of temperature sensors located on the cryogenic structures.

Moreover, it is expected that magnetic $2.5 \mathrm{~T}$ configurations are to be extended not only to several reference magnetic configurations fully analyzed before [4] (see Table I) but also to many other regimes proposed by physicists with the goal to scan different plasma parameters. Both of these facts are considered in the present preparation activities.

\section{B. Filtering of New Regimes}

At present, the input for the numerical analyses is a set of coil group currents and indication of the induction on axis. Probably in the future it is possible to get from physicists a set of already prepared electromagnetic load distributions along winding pack axis for engineering considerations.

General strategy for the approval or rejection of a proposal is presented in Fig. 6. First branch is an easy acceptance using $10 \%$ rule, which is safe and allows using already prepared data for MI monitoring. Previous analyses indicate that current levels in neighboring coils and their difference define loads on the support elements and main components. Therefore, the following formulation is in use:

TABLE II NON PLANAR COIL TYPE 2 CASE VON-MISES EQUIVALENT STRESS LEVELS (MEGAPASCALS). COMPARISON OF FE PREDICTION (GM 6.01) WITH MEASUREMENTS FOR CYCLES OF CASE A

\begin{tabular}{|c|c|c|c|c|c|c|c|c|c|c|}
\hline \multirow{2}{*}{ Sensor name } & \multirow{2}{*}{$\begin{array}{c}\mathrm{FE} \\
\text { re- } \\
\text { sults }\end{array}$} & \multicolumn{3}{|c|}{$\begin{array}{l}\text { 14-Jul-2015 } \\
\text { 09:07-09:39 }\end{array}$} & \multicolumn{3}{|c|}{$\begin{array}{l}\text { 14-Jul-2015 } \\
10: 36-13: 40\end{array}$} & \multicolumn{3}{|c|}{ Status* } \\
\hline & & $\begin{array}{l}\text { Measu- } \\
\text { rements }\end{array}$ & Diff & $\%$ & $\begin{array}{l}\text { Measu- } \\
\text { rements }\end{array}$ & Diff & $\%$ & $\mathrm{R}$ & $Q$ & NotR \\
\hline AAB32CY001 & 110 & 120 & 10 & 9 & 120 & 10 & 9 & $x$ & . & . \\
\hline AAB32CY002 & 127 & 152 & 24 & 19 & 152 & 25 & 19 & $x$ & . & . \\
\hline АAB39CY001 & 109 & 84 & -25 & -23 & 84 & -25 & -23 & . & $x$ & . \\
\hline AAB49CY001 & 110 & 176 & 66 & 60 & 177 & 67 & 61 & . & . & $x$ \\
\hline AAB12CY001 & 109 & 102 & -7 & -6 & 103 & -7 & -6 & $x$ & . & . \\
\hline AAB12CY002 & 129 & 226 & 97 & 75 & 226 & 97 & 75 & . & . & $x$ \\
\hline AAB59CY001 & 110 & & 628 & 571 & & 628 & 571 & & & $x$ \\
\hline
\end{tabular}

* Reliable, Questionable, NotReliable 


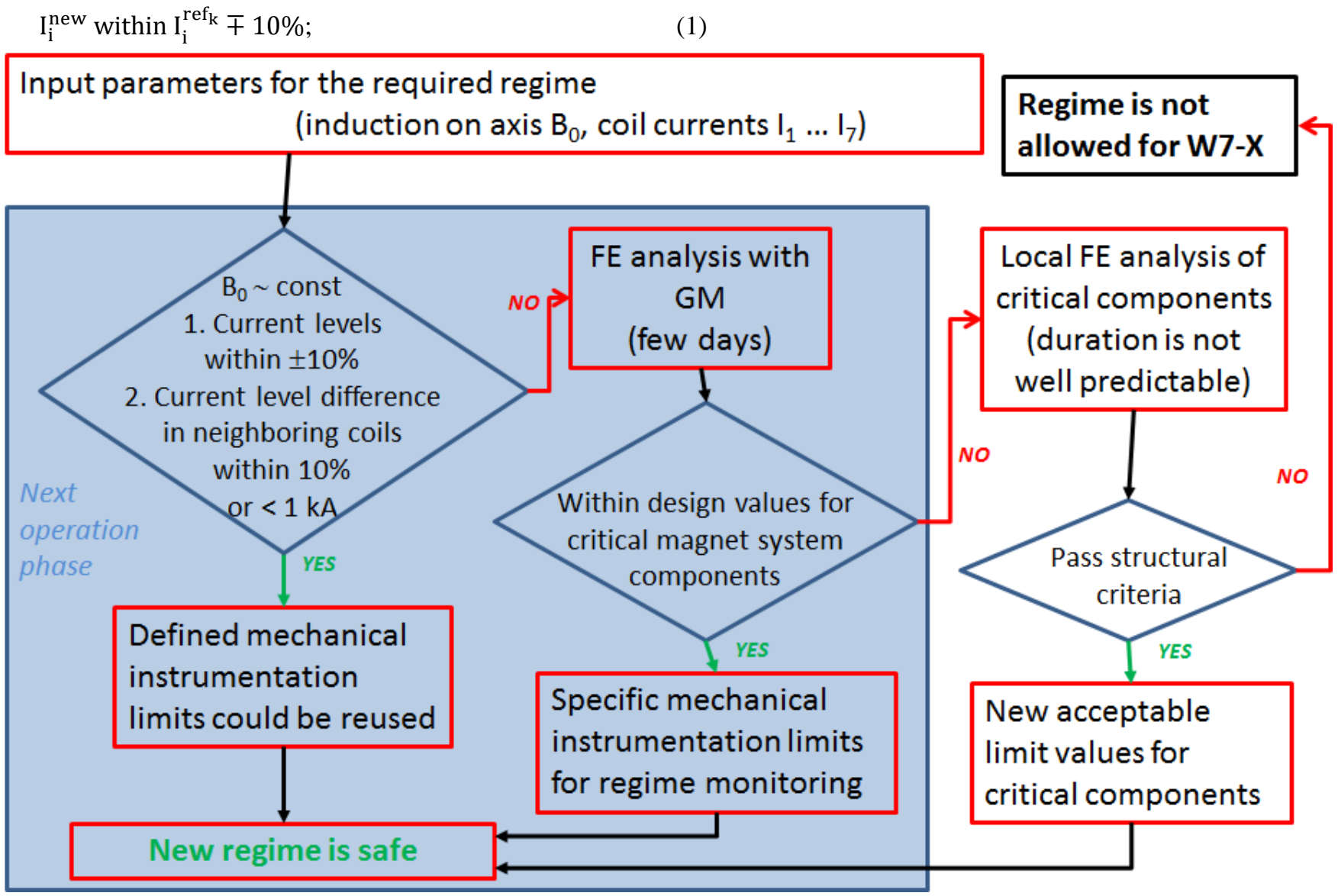

Fig. 6. Filtering of configuration proposals from physicists.

$$
\begin{aligned}
& \mathrm{I}_{j}^{\text {new }} \text { within } \mathrm{I}_{j}^{\mathrm{ref}_{\mathrm{k}}} \mp 10 \% \\
& \left(\mathrm{I}_{\mathrm{i}}-\mathrm{I}_{\mathrm{j}}\right)^{\text {new }} \text { within }\left(\mathrm{I}_{\mathrm{i}}-\mathrm{I}_{\mathrm{i}}\right)^{\text {ref }} \mp(10 \% \text { or } 1 \mathrm{kA})
\end{aligned}
$$

where $I_{i}^{\text {new }}, I_{j}^{\text {new }}$ - currents in neighboring coils defined in a proposal; $I_{i}^{\text {ref }}{ }_{k}, I_{j}^{\text {ref }} f_{k}$ - corresponding current levels in one of reference regime $(\mathrm{k})$.

Only if all conditions (1)-(3) are satisfied, the new configuration is accepted. If that is not the case, FE analysis with GM could deliver the answer: are the generalized forces and moments in all supports and components within design values. If so, new regime is safe, but new limits for MI monitoring are to be prepared as indicated in second branch of the diagram. However, if GM results are not within design values, local analyses of critical components are to be launched. Time period for such complex and sometimes nonlinear elasto-plastic local analysis is difficult to predict, therefore it is agreed during the next phase of operation to permit only regimes satisfying two first branches of evaluations.

Present application of $10 \%$ rule has shown that approximately $30 \%$ of physics proposals could be accepted without additional FE calculations.

\section{Detailed Postprocessing of Cyclic Behavior}

The postprocessing functions of MIViewer have been intensively used to check stable cyclic behavior of the machine. Typical examples of the summary tables for monitored SGs of NPC type 2 are presented in Tables II and III for selected time periods with similar loadings. It is clear visible that for similar cycles during the same monitoring day (Table II), signal results (in blue, italic in all tables) are almost equal, for cycles with a few months span (Table III), they are within a few megapascals. The deviations (in \% and in absolute values) from FE predictions (in black, bold in all tables) are also clear visible there. In order to sort them out and to highlight, status columns are in use: reliable (deviations are below $20 \%$ and values above thresholds), questionable (deviations are below $30 \%$ or values below thresholds) and nonreliable (for other cases).

It is also well visible that a failure occurred in the sensor installed on coil number 59 (AAB59CY001, marked in red). Initially, good results from July 6, 2015 (Table III) jump to extreme value one week later (July 14, Table 
II). Due to the fact that other sensors in the vicinity showed no deviations, the sensor was ranged as malfunctioning one together with other $\sim 5 \%$ MI sensors.

It is planned to use such kind of fast overview reports for repetitive cycles of case $\mathrm{J}$ and case $\mathrm{A}$ at the beginning of new operation phase in order to confirm stable MS behavior after more than one year period including W7-X upgrade activities.

Questionable and nonreliable sensor locations indicated in postprocessing tables for all components were checked in the FE model to confirm their adequate representation as reported in Section IV-E.

Another application of monitoring functions is an overview study for the whole campaign. Highly prestressed Inconel bolts instrumented as shown in Fig. 4 and described in [5] have been carefully checked. Some prestress losses have been found during operation cycles after fast discharges of the MS and after long standby mode. The average rate is approximately $0.1 \mathrm{MPa}$ per ten operation cycles for most critical M30 bolts indicated by sensor AAD10HG611CY9 and AAD20HG611CY7.

As a result, the expected bolt prestress losses during the next operation phase are to be within $13 \mathrm{MPa}$.

Additional attention is paid to the bolt prestress degradations due cooldown/warming up cycle. The values are limited to 14.2 MPa as listed in Table IV for first cooldown cycle. Losses caused by next cooldown/warming up cycle are difficult to predict due to insufficient statistics. During tightening process the bolts have been retightened several times to guarantee that reduction of bolt preloads during specified periods are not above $10 \%$ of the target values at the end of the assembly.

Such approach is absolutely necessary due to the fact that there are no procedures developed either for checking the bolt pretension or for the retightening of the bolts. In spite of the fact that $30 \%$ bolt pretension degradation (200 MPa pretention loss for M30 bolts) has been considered in the parametric FE verification calculations as possible and acceptable event, the close supervision of the bolts is to be extended during next commissioning/operation steps.

Bolt prestress degradation behavior is also observed in the summary tables for cycles (similar to Table III).

\section{Monitoring Improvements}

The monitoring improvements are being implemented in two main directions: 1) to include better interpretation of the signals from the sensors (e.g., temperature compensation as described below) and 2) to accelerate a process of signal extractions, their comparison with three levels of criticality and/or to indicate location of the critical sensors. Full list of improvements includes the followings.

1) Change of strategy for data extraction and storage: daemon process runs and collects required raw data, while postprocessing of meaningful values are delivered only on request.

2) Zeroing stream is organized and written in automatic manner. Zeroing values are updated after each coil energizing start. The software allows also a possibility to have required offset, which is important for longperiod overviews (e.g. for cryolegs and bolts during cooldown).

TABLE IV BOLT PRETENSION OFFSET DUE TO COOLDOWN/WARMING UP CYCLE (MEGAPASCALS)

\begin{tabular}{lc}
\hline \hline Bolt sensor name & Bolt pretention offset, MPa \\
\hline AAD10HG611CY7 & 1.66 \\
AAD10HG611CY8 & -3.65 \\
AAD10HG611CY9 & -9.97 \\
AAD20HG611CY7 & -3.89 \\
AAD20HG611CY8 & -6.04 \\
AAD20HG611CY9 & -14.19 \\
AAD30HG611CY7 & 1.99 \\
AAD30HG611CY8 & -3.41 \\
AAD30HG611CY9 & -6.96 \\
\hline
\end{tabular}

3) Easy review on selected sensor locations is to be completed.

4) Additional sensors to monitor in parallel (mainly temperature: in-vessel components and thermal insulations).

5) Ramping up with simultaneous monitoring and structural assessment is developed and to be tested.

6) Temperature compensation with Kalman filter prediction (see [9] for details) is to be fully tested.

\section{E. FE Global Model Improvements}

The "workhorse" of the engineering team is a MS global $72^{\circ}$ FE model (v.6) prepared in ANSYS. The main improvements are presented in [9] for v.6.01, while recent activities have been devoted to the introduction of model modifications in order to deliver more accurate signal predictions (v.6.02). The driving factor was the plan to double the current levels in planar coils during the next operation phase (see Table I). 
The resulting forces/moments in the relevant components should be higher by factor of $2-3$. Therefore, a campaign to clarify several discrepancies between FE model predictions and measurements as well as a few parametric studies to see their influences on the monitoring signals has been launched with the main focus on PLCs and PSEs.

Two main reasons for discrepancies have been identified:

1) distances between SG positions and nodes in use for FE result extractions and 2) inaccurate assumption about measuring wire inclination. Typical example of the required mesh refinement for fixed support of planar coil type B (PSE B1) is presented in Fig. 7, and corresponding deviation reductions (from GM 6.01 to GM 6.02) are listed in Table $\mathrm{V}$.

The correction of wire inclination as indicated in Fig. 8 has different effects on different mutual coil displacement predictions (see Table VI), but main discrepancy (highlighted in yellow) is gone and the deviations after modifications are in general within $1.1 \mathrm{~mm}$ and below $20 \%$.

Next version of the ANSYS GM (v.6.03) with deviations between all specified sensor positions and FE result extraction nodes below $20 \mathrm{~mm}$ is presently in preparation for the next phase operation.

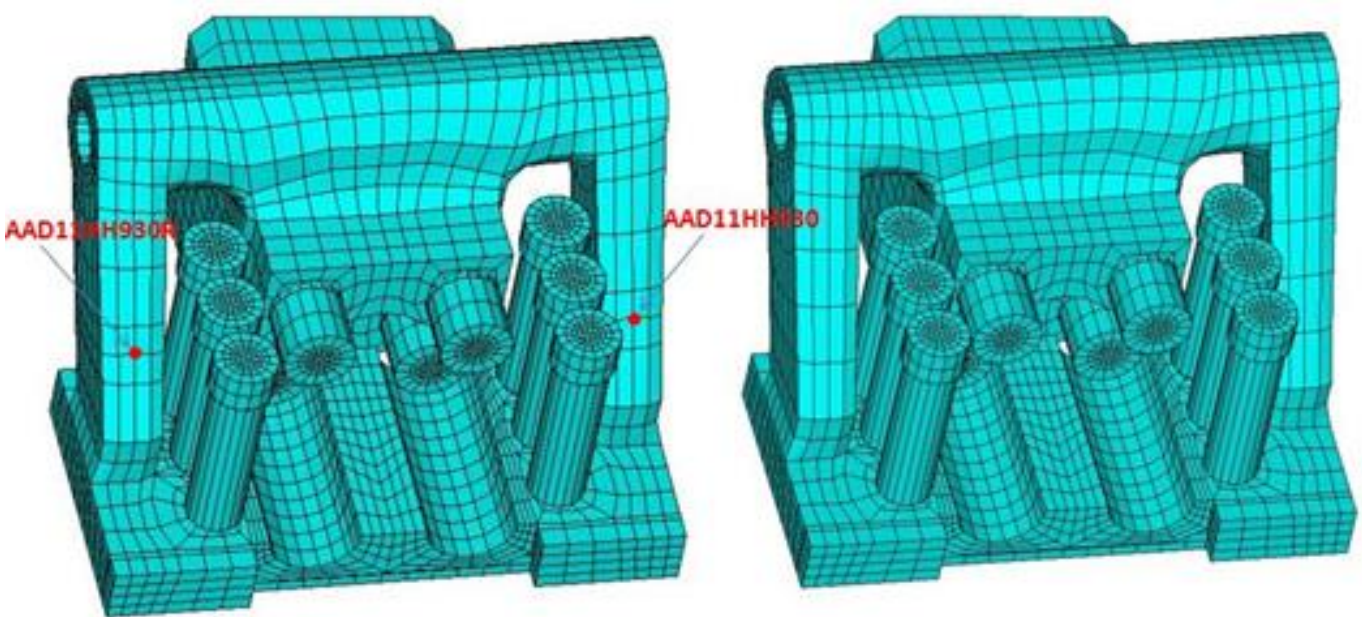

Fig. 7. Refinement of bolted planar coil support B1 for better FE prediction of SG signal (right: original mesh and left: refined mesh).

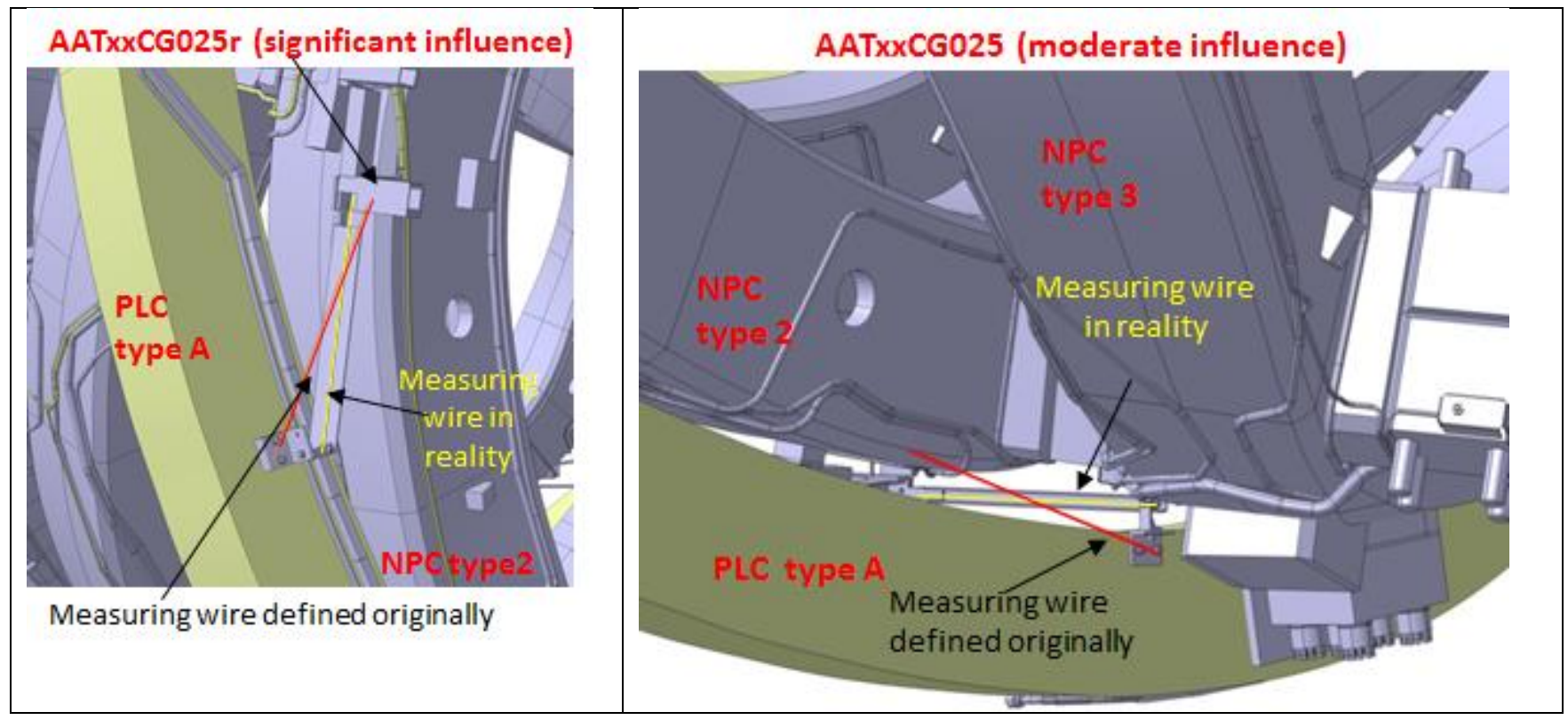

Fig. 8. Typical discrepancies between originally assumed measurement wire inclinations and installed ones. 


\section{STATUS}

TABLE V IMPROVEMENT OF SIGNAL PREDICTION AFTER REFINEMENT OF FE MESH (SEE FIG. 7)

\begin{tabular}{lccc}
\hline \hline \multirow{2}{*}{ Sensor name } & \multicolumn{3}{c}{ Case J (2.5T), MPa } \\
\cline { 2 - 4 } & Original GM 6.01 & Measurements & Refined GM 6.02 \\
\hline AAC52CY002 & $\mathbf{1 8 . 0}$ & $22-24 ;$ & $\mathbf{2 0 . 0}$ \\
AAD30HH930R & $\mathbf{1 2 . 0}$ & 24.2 & $\mathbf{3 0 . 0}$ \\
& & Case A (2.5T), MPa & \\
AAD10HH930 & $\mathbf{2 0 . 0}$ & 62.0 & $\mathbf{4 0 . 0}$ \\
AAD11HH930 & $\mathbf{2 4 . 0}$ & 46.6 & $\mathbf{3 5 . 0}$ \\
AAD31HH930 & $\mathbf{2 4 . 0}$ & 51.8 & $\mathbf{3 5 . 0}$ \\
\hline \hline
\end{tabular}

The successful TC commissioning with a full current allows the use of the coils as a very powerful tool for the TABLE VI IMPROVEMENT OF SIGNAL PREDICTION AFTER REFINEMENT OF WIRE INCLINATION IN FE MODEL (SEE FIG. 8)

\begin{tabular}{|c|c|c|c|c|c|c|}
\hline \multirow{2}{*}{ Sensor name } & FE results & & $\begin{array}{l}\text { Measu } \\
\text { ne cycle }\end{array}$ & $\begin{array}{l}\text { ment } \\
\text { as in Fig }\end{array}$ & & \multirow{2}{*}{$\begin{array}{l}\text { FE results } \\
\text { GM } 6.02\end{array}$} \\
\hline & GM 6.01 & 1 & 2 & 3 & 4 & \\
\hline AAT10CG021 & $-1,67$ & $-2,48$ & $-1,62$ & $-2,16$ & $-2,64$ & $-1,59$ \\
\hline AAT10CG022 & 8,14 & 8,42 & 7,94 & 8,14 & 8,06 & 8,10 \\
\hline AAT10CG023 & $-1,60$ & $-1,63$ & $-1,76$ & $-1,62$ & $-1,39$ & $-1,88$ \\
\hline AAT10CG024 & $-3,41$ & $-2,42$ & $-2,57$ & $-2,61$ & $-2,52$ & $-2,57$ \\
\hline AAT20CG025 & $-10,81$ & $-11,12$ & $-10,33$ & $-10,55$ & $-10,59$ & $-9,77$ \\
\hline AAT10CG025r & -6.00 & $-3,96$ & $-3,73$ & $-3,86$ & $-3,88$ & $-4,04$ \\
\hline AAT10CG026 & $-0,57$ & $-1,27$ & $-1,61$ & $-1,24$ & $-1,26$ & $-0,52$ \\
\hline
\end{tabular}

magnet field adjustment during coming OP1.2a operation. The TC commissioning has been accompanied by displacement measurements and assessment of rubber pad behavior in the coil supports. The collection and analysis of the MI signals are limited by number of expensive signal transformers needed to connect the sensors to the W7-X programmable logic controller system.

The selection of sensors to be connected is done in favor of highly loaded bolts and planar coils, but selection task is to be repeated prior to each step of the commissioning and operation. Few additional electronics have been purchased to reduce number of electronics replug-ins.

It is expected that additional useful information will be collected during OP1.2a phase to minimize deviations for relevant sensors further with probabilistic parameter adjustment in FE models.

The agreement for the next operation phase could be summarized as the followings.

1) Slow current ramping up to test monitoring procedure with the simultaneous MI assessment.

2) Only electromagnetic regimes satisfying criteria without FE local analysis are accepted.

3) Temperature compensation for SGs is to be introduced and checked.

4) Level of current and number of fast discharges for test purposes are to be reduced.

5) Regular assessment of the bolt preload degradation is to be performed. 


\section{CONCLUSION}

The following conclusions can be drawn from recent activities prior to next W7-X operation phase.

1) Results of comparison between numerical modeling and MI measurements show good agreement after introduced modifications in the numerical models.

2) Areas of most attention are defined.

3) Temperature compensation procedure is developed and is to be tested in order to be fully functioning during most demanding operation phases.

4) Approach for fast approval of an extension of physics program is developed.

\section{REFERENCES}

[1] T. S. Pedersen et al., "Confirmation of the topology of the Wendelstein 7-X magnetic field to better than 1:100,000," Nature Commun., vol. 7, Nov. 2016, Art. no. 13493.

[2] T. S. Pedersen et al., "Key results from the first plasma operation phase and outlook for future performance in Wendelstein 7-X," Phys Plasmas, vol. 24, no. 5, p. 055503, 2017.

[3] M. Krychowiak et al., "Overview of diagnostic performance and results for the first operation phase in Wendelstein 7-X (invited)," Rev. Sci. Instrum., vol. 87, no. 11, p. 11D304, 2016

[4] V. Bykov et al., "Specific features of wendelstein 7-X structural analyses," IEEE Trans. Plasma Sci., vol. 42, no. 3, pp. 690-697, Mar 2014..

[5] V. Bykov, "Wendelstein 7-X mechanical instrumentation system for commissioning and operation," Fusion Sci. Technol., vol. 68, no. 2, pp. 267-271, Sep. 2015.

[6] P. van Eeten et al., "Monitoring of W7-X cryostat commissioning with cryostat system FE model," Fusion Eng. Des., vol. 123, pp. 91-96, Nov. 2017

[7] T. Andreeva et al., "Influence of deviations in the coil geometry on Wendelstein 7-X plasma equilibrium properties," Fusion Eng. Des.,vol. 123, pp. 129-132, Nov. 2017.

[8] V. Bykov, J. Fellinger, K. Egorov, F. Schauer, M. Köppen, and H. Jenzsch, "Sliding weight supports for W7-X magnet system Structural aspects," Nucl. Fusion, vol. 55, no. 5, p. 053002, 2015.

[9] V. Bykov, "Engineering challenges of W7-X: Improvement of numerical modeling and mechanical monitoring after commissioning and first phase of operation," Fusion Sci. Technol., vol. 72, pp. 546-558, Nov. 2017.

[10] J. Fellinger, K. Egorov, V. Bykov, and F. Schauer, "Preparation for commissioning of structural sensors of Wendelstein 7-X magnet system,” Fusion Eng. Des., vols. 98-99, pp. 1048-1052, Oct. 2015.

[11] V. Bykov et al., "Structural analysis at the transition from W7-X construction to operation," IEEE Trans. Plasma Sci., vol. 44, no. 9 , pp. 1722-1730, Sep. 2016

[12] A. Carls et al., "A structural integrity monitoring tool for Wendelstein 7-X," presented at the During SOFT Conf., 2016.

[13] K. Risse et al., "First operational phase of the superconducting magnet system of Wendelstein 7-X," Fusion Eng. Des., vol. 124, pp. 10-13, Nov. 2017

[14] H.-S. Bosch, "Engineering challenges in W7-X: Lessons learned and status for the second operation phase," IEEE Trans. Plasma Sci., to be published. 